\title{
Desencuentro de los periodistas con YouTube
}

\section{The disagreement of journalists with YouTube}

\author{
Sonia Blanco; Bella Palomo
}

Cómo citar este artículo:

Blanco, Sonia; Palomo, Bella (2019). "Desencuentro de los periodistas con YouTube". El profesional de la información, v. 28, n. 4, e280411.

https://doi.org/10.3145/epi.2019.jul.11

Artículo recibido el 10-11-2018

Aceptación definitiva: 02-04-2019

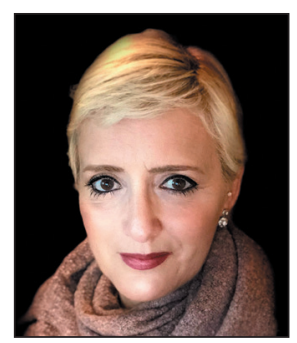

Sonia Blanco

http://orcid.org/0000-0002-1342-7455

Universidad de Málaga

Departamento de Comunicación

Audiovisual y Publicidad

Campus de Teatinos, s/n.

29071 Málaga, España

sblanco@uma.es

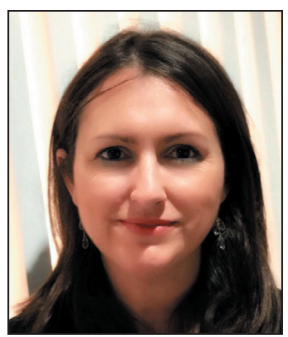

Bella Palomo $\square$

http://orcid.org/0000-0003-2228-5716

Universidad de Málaga

Departamento de Periodismo

Campus de Teatinos, $\mathrm{s} / \mathrm{n}$.

29071 Málaga, España

bellapalomo@uma.es

\section{Resumen}

YouTube, propiedad de Google, es el segundo buscador del mundo. El incremento del consumo online de vídeos ha provocado una reacción en la industria periodística ante la necesidad de conectar con una audiencia que demanda constantemente contenidos audiovisuales. Esta investigación exploratoria demuestra que aunque el vídeo se ha convertido en una prioridad para medios de comunicación y plataformas sociales como Facebook, en España son escasos los periodistas que toman la iniciativa de adoptar un rol activo en la principal plataforma de difusión audiovisual. Del análisis de los canales activos se concluye que el protagonismo del periodista promocionando su perfil profesional en YouTube es reducido, y que la mayoría de sus cuentas ignoran, alteran o rediseñan su actividad laboral habitual.

\section{Palabras clave}

Periodismo; Periodistas; YouTube, Vídeo online; Comunicación audiovisual; Marca personal; Redes sociales; Medios sociales; Confianza; Compromiso; Engagement.

\begin{abstract}
YouTube, owned by Google, is the second search engine in the world. The increase in online video consumption has provoked a reaction in the journalistic industry to attend the need to connect with an audience that constantly demands audiovisual content. This exploratory research shows that although video has become a priority for media and social platforms such as Facebook, in Spain there are few journalists who take the initiative to take an active role in the main video sharing platform. From the analysis of active channels developed by reporters, we conclude that the role of the journalist promoting his/her professional profile on YouTube is scarce, and that most of his/her accounts ignore, alter or redesign his usual work activity.
\end{abstract}

\section{Keywords}

Journalism; YouTube; Online video; Audiovisual communication; Personal brand; Social networks; Social media; Trust; Engagement; Journalists.

Financiación

Investigación financiada por el proyecto del Ministerio de Economía y Competitividad: La influencia de la audiencia en la innovación periodística y gestión de la participación: riesgos y oportunidades (CSO2015-64955-C4-3-R). 


\section{Introducción: nuevos hábitos, nuevos compromisos}

Desde 2012 el consumo de las redes sociales en España supera a la lectura de noticias de actualidad en cibermedios (EGM, 2012), pero nada ensombrece el interés de la audiencia por los contenidos audiovisuales. La televisión sigue siendo el medio tradicional de mayor penetración, y dos terceras partes de los jóvenes españoles entre 18 y 29 años aseguran ver las noticias emitidas por esa vía diariamente (Matsa et al., 2018). Una expectación aún mayor se ha forjado en torno a los contenidos audiovisuales online. Con más de 25 millones de visitantes únicos al mes, YouTube es el sitio de internet de mayor éxito en España

Con más de 25 millones de visitantes únicos al mes, YouTube es el sitio de internet de mayor éxito en España

(EGM, 2018) y el segundo buscador del mundo después de Google. Su consumo, junto al de Facebook y WhatsApp, posee además una influencia directa en la dieta informativa de los ciudadanos (Kalogeropoulos, 2017), como apunta el Digital news report.es 2018 (Amoedo; Vara-Miguel; Negredo, 2018). Según dicho informe, el 47\% de los jóvenes entre 18 y 34 años utiliza Facebook para conocer la actualidad, y en el caso de YouTube el 32\% visita la plataforma para ver, comentar y compartir noticias.

Esta tendencia, clonada en el plano internacional (Ericsson Consumerlab, 2017), refleja la evolución de la construcción social del conocimiento, donde la expansión de Instagram y Snapchat entre los jóvenes también confirma el éxito del vídeo online en la actualidad (Smith; Anderson, 2018). La imagen permite corroborar hechos y, por lo tanto, influye en la credibilidad del medio en un período de desconfianza caracterizado por la circulación de noticias falsas (Wardle; Derakhshan, 2017; Ireton; Posetti, 2018). Conscientes de esta realidad y ante la necesidad de conectar con la audiencia (Masip, 2016) aplicando estrategias basadas en la proximidad y la transparencia (Harbers, 2016), los medios han reaccionado aumentando su oferta de contenidos audiovisuales (Kalogeropoulos, 2017) e invirtiendo en nuevas narrativas como el periodismo inmersivo (Benítez-De-Gracia; Herrera-Damas, 2018). Aunque algunos expertos han puesto en duda sus beneficios (Banikarim,

El consumo de YouTube posee una influencia directa en la dieta informativa de los jóvenes

2017), incluso Facebook ha pagado a las empresas editoras que han emitido vídeos en directo en su plataforma (Jackson, 2016), lo que demuestra la influencia que estas empresas tecnológicas poseen en la configuración de la oferta de los formatos informativos.

De las plataformas de vídeo, YouTube es hoy el líder del mercado con más de mil millones de usuarios y cientos de millones de horas de vídeo visionadas al día (Berrocal-Gonzalo; Martín-Jiménez; Gil-Torres, 2017), datos que seguirán creciendo porque se estima que en 2019 el 80\% del tráfico mundial de internet se dirigirá fundamentalmente a contenidos audiovisuales (Serrano-Cobos, 2016). Considerada la segunda red que más ha impactado en los medios de comunicación (Campos-Freire et al., 2016), paradójicamente existen evidencias de que en las agendas mediáticas YouTube protagoniza menos titulares en comparación con los que logran Facebook o Twitter (Paulussen; Harder, 2014).

Para reducir este desencuentro, y conscientes de la elevada producción amateur que alojan y de que las principales búsquedas que realizan los usuarios en YouTube siguen relacionadas con la música, los tutoriales y los videojuegos, la plataforma está fomentando la profesionalización de la actividad (De-Aguilera-Moyano; Castro-Higueras; Pérez-Rufí, 2019) y, concretamente, en 2018 incrementó su compromiso con el periodismo de calidad promocionando fuentes informativas con autoridad y recomendando el consumo de dichos vídeos para combatir la desinformación (Lapowsky, 2018).

\section{Diseñando un perfil híbrido}

La presencia de nuevos actores y nuevos hábitos configura un escenario comunicativo híbrido y complejo de transformaciones drásticas y constantes, donde el periodismo adaptativo (Palomo; Palau-Sampio, 2016) es un requisito imprescindible para su supervivencia y justifica la realización de un estudio crítico que recoja la respuesta del profesional de la información a dichos cambios y detecte tendencias de futuro.

Para los medios ha supuesto un reto abandonar su relación directa con la audiencia (Noguera-Vivo, 2016), descentralizar su producción y diseminar contenidos en canales como YouTube (Peer; Ksiazek, 2010), porque los productos ahí alojados no le reportan tráfico a sus webs, situación que provocó que Mediaset rechazara durante ocho años el servicio de la plataforma. Actualmente la mayoría de los canales de televisión tradicionales españoles alojan vídeos en YouTube, destacando el medio millón de suscriptores de Canal Sur, cifra que supera al resto de las cadenas. El país también es un referente por la oferta diversificada que realiza en su canal, incluyendo vídeos de 360o, reportajes audiovisuales, vídeos virales, y La Voz de Iñaki, sección de periodismo de opinión protagonizada por Iñaki Gabilondo.

Para los periodistas, la transición de la práctica profesional a las redes sociales tampoco se ha producido con la misma intensidad en las diferentes plataformas. Por este motivo, estudios previos sobre la representación de la identidad pro- 
fesional y personal del periodista en medios sociales se han centrado casi exclusivamente en Twitter (Hanusch; Bruns, 2017; Molyneux, 2014) aunque algunos de sus resultados pueden ser extrapolables a otras plataformas. Investigadores como Bossio y Sacco (2017) advierten que los periodistas pueden representar tres tipos de identidades: la identidad transicional, la identidad de marca y la identidad social, influidas por el trasvase de normas tradicionales como la objetividad al entorno online, la presión de las empresas periodísticas para que su personal extienda su influencia a internet, y la búsqueda de un tono más personal y auténtico, a veces basado en la mezcla de humor y opinión, que favorezca la participación ciudadana. Las tres modalidades son complementarias e influyen en la marca personal del periodista (Marauri-Castillo; Cantalapiedra-González; Álvarez Fernández, 2018; Chen, 2013).

En esa línea, Origgi (2018) sostiene que estamos "finalizando la era de la información para dar paso a la era de la reputación", y que ésta actúa como el nuevo gatekeeper del conocimiento y obliga a monitorizar y cuidar constantemente la presencia digital. Desde el Nieman Lab también se apuntó como predicción para 2019 la expansión del periodista-influencer creador de podcasts independientes o un canal en YouTube (Lorenz, 2018).

Pero a pesar de estos vaticinios, el emprendimiento en redes sociales de los periodistas se ha ligado también a un incremento de la tensión (Lewis, 2012), de la ansiedad, y al sacrificio o la pérdida de la identidad personal online (Holton; Molyneux, 2017) de quien ha estado habituado al anonimato profesional. Por ello, la interpretación y el análisis tienden a ser sutiles (Molyneux, 2014) para evitar polémicas, la acción de los haters y distanciarse de prácticas autopromocionales que se vinculan más al terreno del marketing (Tandoc; Vos, 2015) que al del periodismo tradicional.

\section{Objetivos y metodología}

En este contexto, nos preguntamos qué iniciativas han lanzado los periodistas en YouTube sin estar asociadas o respaldadas por un medio de comunicación. A pesar del evidente giro conceptual de la plataforma, de las posibilidades que ofrece y el impacto generado en las rutinas de las redacciones, las escasas investigaciones que han abordado esa cuestión han analizado casos de periodismo alternativo o ejemplos de periodismo ciudadano (Antony; Thomas, 2010). Sin embargo, sí se detecta una preferencia por analizar el impacto que YouTube ha tenido sobre otra figura, la del político.

Para averiguar cómo se han adaptado los profesionales de la información al principal portal de vídeos en internet, los objetivos complementarios de esta investigación consisten en examinar cuantitativamente los canales en YouTube creados a iniciativa de periodistas, analizar las características de estos espacios y categorizar sus perfiles de participación. Para alcanzar los objetivos descritos se ha planteado una aproximación multimetodológica.

En una primera fase llevada a cabo en enero de 2018 se diseñó una base de datos de periodistas activos en redes sociales en España en colaboración con la empresa de ingeniería analítica Alianzo. De los 1.131 perfiles profesionales detectados, sólo 243 incluían YouTube entre sus redes.

Un análisis preliminar permitió detectar que el concepto de "periodista" que maneja Alianzo resulta laxo al incluir numerosos usuarios que no eran periodistas por formación ni por ocupación, debilidad detectada por investigaciones previas en otras bases de datos similares (Molyneux; Lewis; Holton, 2018). Con objeto de dotar de rigurosidad a la muestra seleccionada, en una segunda fase se comprobaron los perfiles del millar de usuarios en Twitter, Facebook, Linkedln, e incluso se contactó vía correo electrónico, redes sociales y teléfono, lo que también permitió detectar estrategias específicas de relación con la audiencia en algún caso. El ámbito de la comunicación es dinámico y complejo, por lo que en esta fase se comprobó que los usuarios atendían a seis perfiles:

- Licenciado o graduado en Periodismo o Comunicación con actividad profesional activa en dichos ámbitos.

- Licenciado o graduado en Periodismo o Comunicación que actualmente no ejerce la profesión.

- Profesional de medio de comunicación sin formación periodística o similar.

- Licenciado o graduado de otra disciplina, pero con master u otra formación superior relacionada con la comunicación.

- Especialista en algún área, responsable de un blog o que escribe regularmente en algún medio, pero no es su actividad profesional principal.

- Colaborador ocasional en medios de comunicación.

Este contexto obligó a aplicar dos nuevos filtros para la selección de la muestra final:

- aquellos usuarios formados en periodismo y/o comunicación, que se dedican o se han dedicado profesionalmente a esta actividad;

- profesionales con una dilatada trayectoria en medios de comunicación, aunque no posean estudios periodísticos previos.

Estos criterios redujeron el listado a 175 perfiles. De ellos 30 nunca habían subido un vídeo a su canal de YouTube, frente a 145 que sí habían publicado contenido audiovisual en dicha plataforma. Este directorio se actualizó en una tercera fase con otra búsqueda filtrada en YouTube y Google, añadiéndose 62 perfiles más de periodistas youtubers, independientemente de que el contenido estuviese vinculado o no a su actividad profesional habitual. En total, se construyó un directorio de 207 profesionales de la información.
Los medios siguen dedicando menos titulares a YouTube, en comparación con Facebook y Twitter 
La heterogeneidad detectada en su actividad en la plataforma ha obligado a aproximarnos al fenómeno analizando dos grupos:

- periodistas activos en YouTube que obtienen las mejores métricas, y

- quienes ocupan las últimas posiciones.

En ambos casos el requisito mínimo era que hubiesen actualizado su canal con posterioridad al 1 de enero de 2017, ya que aunque los contenidos antiguos siguen posicionando en Google, esta investigación pretende realizar una radiografía del periodista youtuber en la actualidad. Además, para diseñar ambos grupos fijamos tres criterios relevantes, debiendo cumplir obligatoriamente al menos dos de ellos:

- número de vídeos subidos: determina la relevancia que el periodista otorga a la plataforma en su estrategia de comunicación;

- número de suscriptores: refleja la fidelización del canal;

- número de visualizaciones: muestra el desempeño y el éxito de los vídeos.

Esta aproximación cuantitativa general se complementó con un análisis cualitativo de la producción periodística generada por los periodistas youtubers activos con las mejores métricas y, por tanto, con mayor éxito. Se seleccionaron 102 vídeos para la muestra, con una duración que oscila entre los 12 segundos y los 72 minutos, atendiendo a estos criterios:

Tabla 1. Criterios aplicados para determinar los perfiles de la muestra

- sus tres primeros vídeos;

- los tres vídeos más recientes;

- aquel con un mayor número de reproducciones, si no estaba entre los anteriores.

Para el análisis audiovisual la entrada de datos fue asistida por un formulario testado en dos ocasiones para asegurar su fiabilidad, contemplando los siguientes parámetros: formatos, géneros, temáticas, estructuras narrativas, rótulos, relación con la audiencia, estrategias de convergencia periodística, estructura del discurso, intencionalidad del mensaje; aspectos técnicos como el tipo de edición de planos usados, el sonido, la música, la iluminación; y elementos semánticos como el título del vídeo y la descripción.

Desde la perspectiva de la participación, se analizaron los comentarios, distinguiendo las reacciones negativas y positivas a los vídeos publicados.

\section{Resultados}

\subsection{Niveles de participación del periodista en YouTube}

El estudio permite categorizar la actividad del periodista en YouTube distinguiendo dos grandes perfiles:

- periodistas creadores de contenidos específicos para su canal;

- periodistas que utilizan la plataforma como repositorio.

Esta segunda modalidad, de carácter menos creativo, presenta también distintas opciones:

- profesionales que almacenan sus apariciones televisivas y/o conferencias impartidas;

- periodistas que exponen vídeos vinculados a sus intereses personales que no son de producción propia ni están relacionados con su actividad laboral, y que en la mayoría de las ocasiones carecen de derechos sobre la pieza (fragmentos de películas, fotomontajes de artistas reconocidos, piezas televisivas...);

- periodistas que publican brutos o fragmentos de su trabajo con carácter previo o posterior a su emisión en el medio convencional;

- una última modalidad híbrida y sin una finalidad definida que combina las opciones previas con material de índole personal de producción propia (viajes privados, celebraciones personales, montajes fotográficos familiares).

Sin embargo, el análisis demuestra la imposibilidad de establecer un único patrón de comportamiento del periodista youtuber de éxito porque las métricas alcanzadas son muy heterogéneas. Como se observa en la tabla 2, sí podemos confirmar que sólo 36 perfiles estarían aprovechando adecuadamente las posibilidades de YouTube en España, aunque sólo el $41,6 \%$ de ellos crean contenidos periodísticos. En este grupo el promedio de suscriptores es de 50.821 , con un amplio rango que va desde los 71 hasta los 786.699. La media de vídeos subidos es de 260, aunque los extremos oscilan entre los 11 y los 2.675. El promedio de reproducciones totales roza los seis millones,

Hay dos tipos de periodistas en YouTube: los que crean contenidos específicos para su canal y quienes utilizan la plataforma como repositorio 
pero resulta especialmente llamativo el caso de Luis Calabor, que duplica esta cifra con más de 12 millones de reproducciones, sólo con 356 vídeos publicados y 6.944 suscriptores. Este rendimiento excepcional demuestra que no hay una relación directa entre los suscriptores y las reproducciones. De hecho, un ejemplo inverso es el de Rodrigo Fáez, cuyo canal posee 65.220 suscriptores, pero apenas supera el millón y medio de reproducciones. En esa misma línea, el canal de Iker Jiménez, popular periodista por sus programas televisivos y radiofónicos, atrae a muchos suscriptores, aunque ello no garantiza que los vídeos sean los más vistos. Un elevado número de vídeos subidos tampoco se acompaña necesariamente de más visualizaciones, ya que Fernando Berlín casi cuadruplica en publicaciones a Calabor (1.200 vídeos) y no llega a los dos millones de reproducciones, probablemente influido porque en realidad predominan los archivos de audio de La cafetera, y el grueso de oyentes de podcasts está en otras plataformas más específicas como iTunes o iVoox.

Tabla 2. Grupo A. Periodistas en YouTube con mejores resultados en sus canales

\begin{tabular}{|c|c|c|c|c|c|c|}
\hline Apellidos & Nombre & Suscriptores & N. vídeos & Visualizaciones & $\begin{array}{c}\text { Antigüedad } \\
\text { del canal }\end{array}$ & Empresa \\
\hline Arráez & David & 929 & 69 & 179.745 & $16-2-19$ & El cyberdiario \\
\hline Berlín & Fernando & 5.936 & 1.200 & 1.856 .897 & $3-5-06$ & Radiocable.com \\
\hline Cabrera & Andrés & 19.192 & 74 & 1.033 .285 & $8-3-16$ & Kaiser magazine \\
\hline Calabor & Luis & 6.944 & 356 & 12.562 .047 & $20-6-07$ & El correo \\
\hline Carretero & Manuel & 513 & 296 & 524.224 & $3-10-11$ & Canal Sur TV \\
\hline Casquet & Noemí & 18.574 & 22 & 1.228 .208 & $29-12-12$ & Freelance \\
\hline Coria & Javier & 614 & 11 & 340.412 & $22-11-08$ & $\mathrm{Ns} / \mathrm{Nc}$ \\
\hline Dadá & Irina & 33.856 & 122 & 2.734 .551 & $14-2-11$ & $\mathrm{Ns} / \mathrm{Nc}$ \\
\hline Del Río & Modesto & 71 & 116 & 238.838 & $28-1-06$ & Radio galega \\
\hline Durán Escabias & Imanol & 684 & 64 & 660.423 & $21-5-06$ & RTVE \\
\hline Fáez & Rodrigo & 65.220 & 92 & 1.586 .042 & $16-10-12$ & Bein Sports España \\
\hline Fares & Fátima & 129 & 150 & 179.194 & $10-9-10$ & Prensa Ibérica \\
\hline Gago & Manuel & 508 & 116 & 119.818 & $18-5-10$ & Culturagalega.org|USC \\
\hline Gómez & Lourdes & 1.297 & 175 & 36.651 & $17-1-17$ & Canal Extremadura| Freelance \\
\hline González & María del Amor & 11.516 & 234 & 24.271 .091 & $6-10-07$ & $\mathrm{Ns} / \mathrm{Nc}$ \\
\hline Herreros & Pablo & 293 & 124 & 426.609 & 29-9-06 & Goodwill Comunicación |ISDI \\
\hline Jiménez & Iker & 56.876 & 119 & 2.789 .010 & $25-8-14$ & Cuarto milenio | Alma Productora AV \\
\hline Manfredi & Antonio & 139 & 159 & 266.616 & $17-5-06$ & RTVA \\
\hline Martínez Ron & Antonio & 633 & 93 & 2.328 .201 & $22-11-06$ & Vozpopuli \\
\hline Matallanas & Javier G. & 198 & 56 & 1.467 .589 & $19-11-06$ & Diario As |As TV \\
\hline Muñoz Morales & Silvia & 402.485 & 342 & 34.866 .857 & $28-1-10$ & SoyOlivia.com | Flooxer \\
\hline Ojeda & Álvaro & 46.632 & 335 & 10.826 .965 & $2-6-16$ & OkDiario.com \\
\hline Quevedo & Luis & 4.865 & 132 & 2.368 .480 & $10-9-06$ & Telemadrid \\
\hline Represa & Bea & 6.719 & 124 & 430.925 & $11-10-11$ & Freelance \\
\hline Riesco & Nerea & 295 & 175 & 102.070 & $14-12-08$ & Escritora y freelance \\
\hline Saavedra & Fran J. & 446 & 156 & 117.964 & $26-4-06$ & Radio Esperantia y freelance \\
\hline Salas & Meritxell & 107.614 & 146 & 8.174 .362 & $1-9-14$ & SolidarityApp.com | Qbitapp \\
\hline Salas & Silvia & 786.699 & 277 & 64.486 .041 & $13-9-12$ & Freelance \\
\hline Salvia & Jordi & 802 & 239 & 3.794 .003 & $19-1-06$ & Candidatura d'Unitat Popular \\
\hline Salza (Geekguy) & César & 15.962 & 81 & 384.690 & 21-10-15 & Cenet.com/es \\
\hline San Juan & Valentí & 167.628 & 106 & 13.244 .513 & $25-5-10$ & Freelance \\
\hline Sánchez González & Juan Luis & 314 & 14 & 417.795 & $2-3-11$ & $\mathrm{Ns} / \mathrm{Nc}$ \\
\hline Santos (Chicageek) & Elena & 32.089 & 217 & 2.961 .683 & $4-5-09$ & Freelance \\
\hline Senabre & Dani & 18.306 & 78 & 958.515 & $17-9-16$ & COPE \\
\hline Velarde & Antonio & 14.344 & 2.675 & 10.400 .807 & $6-10-14$ & Periodista Digital.com \\
\hline Villacañas & Javier & 249 & 629 & 1.644 .865 & 14-1-09 & COPE \\
\hline
\end{tabular}

La mayoría de estos perfiles destacados tienen en común que gran parte de sus vídeos son de elaboración propia, y en muchos casos editados y producidos para ser más atractivos. Pero la temática del canal también ayuda. Calabor es fotoperiodista de sucesos, contenido de especial interés para la audiencia (Røssland, 2007), y realiza un uso pragmático de las redes sociales publicando en sus perfiles su número de teléfono con objeto de que cualquier ciudadano pueda 
ofrecerle información. Según declara el periodista, esta estrategia le resulta muy efectiva, y le permite tener la primicia de muchos sucesos.
Sólo tres de cada diez periodistas destacados en YouTube son mujeres

Entre los canales de creación más reciente destaca el de Dani Senabre. Inaugurado en septiembre de 2016, ha alcanzado 18.306 suscriptores y casi un millón de reproducciones. La clave del crecimiento es también su enfoque, ya que está claramente concebido como medio de autopromoción y marca personal.

Tabla 3. Grupo B. Periodistas en YouTube con peores resultados en sus canales

\begin{tabular}{|c|c|c|c|c|c|c|}
\hline Apellidos & Nombre & Suscriptores & $\begin{array}{c}\mathrm{N} . \\
\text { vídeos }\end{array}$ & Visualizaciones & $\begin{array}{c}\text { Antigüedad } \\
\text { canal }\end{array}$ & Empresa \\
\hline Antolín Llorente & Ion & 9 & 23 & 8.319 & $11-9-07$ & Caixabank \\
\hline Barquilla & Fran & 20 & 13 & 32.166 & $13-5-10$ & Grupo Joly | Ideas con Alma \\
\hline Barrientos & Victoria & 20 & 22 & 60.364 & $21-4-06$ & Freelance \\
\hline Basterra & Juanjo & 5 & 22 & 5.141 & $2-2-13$ & Gara \\
\hline Bianchi Galacho & Mar & 673 & 12 & 9.690 & $12-1-17$ & Deltatre | Diario sport \\
\hline Bohórquez & Álvaro & 21 & 74 & 22.952 & $6-8-10$ & A la velocidad absurda (radio) | Freelance \\
\hline Briones & José Luis & 17 & 35 & 57.602 & $20-2-06$ & Freelance \\
\hline Camacho & Gemma & 68 & 35 & 22.983 & $13-9-16$ & España directo (TVE) \\
\hline Chamorro Galán & Macarena & 19 & 27 & 12.847 & $2-7-13$ & Atlético Play | Freelance \\
\hline Crespo & Concha & 28 & 44 & 9.473 & $25-5-12$ & Freelance \\
\hline Cuesta & Consuelo & 0 & 19 & 1.555 & $12-6-08$ & Freelance \\
\hline De Francisco & Tania & 1 & 1 & 101 & $24-1-17$ & CCTV+ | Wall Street International \\
\hline Ferragud & Inma & 7 & 3 & 135 & $23-11-15$ & Consultora freelance \\
\hline Ferrer & Aurora & 7 & 1 & 77 & $15-5-17$ & Freelance \\
\hline García Barahona & Vanessa & 10 & 17 & 1.529 & $15-7-13$ & Syndicali \\
\hline González Zorrilla & Raúl & 3 & 5 & 632 & $28-4-10$ & La tribuna del País Vasco \\
\hline Izaguirre & Ander & 7 & 9 & 2.524 & $31-12-07$ & Freelance \\
\hline Lara & Javier & 38 & 20 & 38.669 & $30-11-12$ & IC Grupo | IES Emilio Muñoz \\
\hline López & Cecilia & 11 & 22 & 59.467 & $27-8-07$ & Associació de Comerciants de la Cerdanya y otros \\
\hline Marco & Vicent & 29 & 14 & 27.040 & $26-5-13$ & VCF Radio|Grupo Secuoya \\
\hline Martín Herrero & Sara & 19 & 16 & 5.350 & $15-1-12$ & RTV Castilla y León \\
\hline Molina & Esther & 16 & 45 & 4.349 & $27-11-14$ & EFE Radio | Freelance \\
\hline Montesinos & Alo & 3 & 76 & 3.141 & 8-10-10 & Consultor freelance \\
\hline Núñez Díaz & Rubén & 29 & 24 & 11.367 & $29-8-12$ & Radio Huétor Tájar \\
\hline Paniagua & Soraya & 8 & 18 & 584 & $1-5-10$ & Consultora freelance \\
\hline Pascual & Cristian & 40 & 7 & 32.230 & $6-9-07$ & Fundación General Universidad de Valladolid \\
\hline Pereiro & Xosé manuel & 11 & 11 & 5.309 & $12-2-07$ & Revista Luzes \\
\hline Pérez Espinosa & Ana Belén & 0 & 7 & 3.301 & $22-9-10$ & Freelance \\
\hline Reig & Marga & 10 & 16 & 8.230 & $10-7-15$ & Canal Sur TV \\
\hline Rey Calatayud & Víctor & 41 & 22 & 17.250 & 24-10-11 & Valencia CF \\
\hline Riveiro & Aitor & 4 & 22 & 14.207 & $12-5-12$ & Eldiario.es \\
\hline Rodríguez & Rosa María & 5 & 16 & 4.348 & $5-5-13$ & IES Clara Campoamor \\
\hline Rull & Antonio & 9 & 62 & 19.179 & $9-9-06$ & Flat 101 \\
\hline Sainz Esteban & Inés & 136 & 38 & 10.738 & $24-6-14$ & Back Up Comunicación y Prensa \\
\hline Sempere & Vicent & 7 & 15 & 2.372 & $22-6-06$ & MediaPro \\
\hline Seseña & Daniel & 19 & 32 & 10.095 & 26-6-09 & TVE \\
\hline Slafer & Tomás & 6 & 25 & 1.325 & $30-9-11$ & Soymotor.com \\
\hline Tadeo & María & 3 & 12 & 162 & 3-11-17 & Bloomberg TV \\
\hline Valero & Elena & 0 & 42 & 2.125 & $5-3-13$ & Freelance \\
\hline Ventura & Borja & 20 & 41 & 274.340 & $31-5-06$ & 30 letras | Freelance | Universidad Carlos III \\
\hline Zaballa & Julen & 45 & 41 & 16.700 & $7-8-06$ & El crisol. Ideas y comunicación \\
\hline Zumeta Landaribar & Gorka & 17 & 9 & 704 & $3-12-17$ & ESIC \\
\hline
\end{tabular}


La evolución del canal en el tiempo también es otra peculiaridad que responde al perfil flexible que se demanda en el periodista actual (Singer, 2014). Valentín San Juan empezó con vídeos cortos como sugieren los expertos (Chi, 2018), pero entre sus piezas más recientes hay documentales de 1 hora, que acumulan casi un millón de reproducciones, cifras de audiencia que se aproximan a las alcanzadas por la televisión tradicional, aunque el tiempo para lograrlo sea mayor. En general, resulta destacable que la duración media de los vídeos más largos en el grupo A sea de 46 minutos, más del doble que en el grupo B, y la justificación es que el periodista refuerza su autoridad y marca personal exhibiendo cursos, conferencias, mesas redondas y programas televisivos donde participa.

En el análisis cuantitativo se observa también un desequilibrio por género, ya que las periodistas youtubers destacadas representan sólo el 30,6\% del listado de profesionales. Este panorama confirma la brecha participativa de la mujer detectada previamente en otras plataformas como Wikipedia (Ford; Wajcman, 2017; Shaw; Hargittai, 2018;), donde menos del $10 \%$ de los editores de los contenidos son mujeres.

También requiere de iniciativa la personalización del url ya que el periodista debe solicitarlo a la plataforma. En la tabla 2, 30 de los 36 perfiles (83,3\%) tienen este rasgo distintivo. La misma relevancia posee la descripción del canal, ya que en cuestiones de SEO (search engine optimization) es mucho más fácil catalogar el texto que el vídeo, por lo que este componente textual es fundamental para posicionar mejor el canal en los buscadores. El 80,6\% de la muestra (29 perfiles) sí incluye dicha descripción.

Con números muy inferiores en cada uno de los apartados, el grupo B (tabla 3) está configurado por aquellos periodistas que prioritariamente utilizan la plataforma como repositorio audiovisual.

La media de suscriptores es sólo de 34, y algunos casos tienen cero seguidores. La media de vídeos almacenados es de 24, en canales activos, cuya antigüedad suele superar los cinco años. Las reproducciones también son muy escasas, con un promedio de 19.540, y en este grupo sólo destaca el periodista Borja Ventura con 274.320 visualizaciones, si bien sus tres vídeos más destacados no son de autoría propia.

Sin embargo, en este grupo la participación desde la perspectiva de género se equipara, con 22 hombres y 20 mujeres. La reducción de las habilidades digitales también se evidencia con menos direcciones personalizadas (66,7\%), el descenso de las descripciones del canal $(42,9 \%)$ y con una apuesta por los vídeos de corta duración, que oscilan entre los 36 segundos y los 22 minutos.

\subsection{Un territorio por explorar periodísticamente}

Como se ha adelantado, de los 36 perfiles de éxito detectados, aun siendo todos periodistas, el 58,4\% no desarrolla contenidos vinculados a las hard news (Reinemann et al., 2011) o a temáticas tradicionalmente periodísticas, y permanecen en una fase experimental creando montajes fotográficos personales, o proyectan una imagen más comercial y próxima a la publicidad como youtubers de belleza (García-Rapp, 2016), la especialidad más destacada.

Este contexto tan diverso obligó a abordar la parte cualitativa seleccionando sólo los perfiles que crean contenidos periodísticos (tabla 4$)$.

Las temáticas predominantes en los vídeos de este grupo son los deportes $(15,7 \%)$, la tecnología $(11,8 \%)$, seguido muy de cerca por sociedad $(10,8 \%)$ y el misterio $(10,8 \%)$. Las estructuras más empleadas son la expositiva $(55,9 \%)$, narrativa $(33,3 \%)$ y, a gran distancia, la argumentativa (5,9\%). El protagonismo del periodista es limitado, ya que su presencia en pantalla se reduce al $30,4 \%$ de la duración del vídeo, aunque en 2 de cada 10 vídeos nunca aparece su imagen. Sólo una cuarta parte de los vídeos no utilizan rótulos, y en el resto predominan los identificativos $(30,4 \%)$ e informativos $(16,7 \%)$.

Respecto a su dimensión social, el usuario tiene un papel secundario en estos canales a pesar de su influencia en la construcción de una identidad online. En el 30,2\% de los vídeos no se visualiza ninguna relación o interacción con la audiencia, un 23,5\% interpela al público normalmente tuteándo-

Tabla 4. Perfiles de periodistas youtuber seleccionados para el análisis cualitativo

\begin{tabular}{|l|l|r|r|r|}
\hline \multicolumn{1}{|c|}{ Apellidos } & \multicolumn{1}{c|}{ Nombre } & Suscriptores & N. vídeos & Visualizaciones \\
\hline Arráez & David & 929 & 69 & 179.745 \\
\hline Cabrera & Andrés & 19.192 & 74 & 1.033 .285 \\
\hline Calabor & Luis & 6.944 & 356 & 12.562 .047 \\
\hline Casquet & Noemí & 18.574 & 22 & 1.228 .208 \\
\hline Durán Escabias & Imanol & 684 & 64 & 660.423 \\
\hline Fáez & Rodrigo & 65.220 & 92 & 1.586 .042 \\
\hline Gago & Manuel & 508 & 116 & 119.818 \\
\hline Jiménez & Iker & 56.876 & 119 & 2.789 .010 \\
\hline Manfredi & Antonio & 139 & 159 & 266.616 \\
\hline Martínez Ron & Antonio & 633 & 93 & 2.328 .201 \\
\hline Quevedo & Luis & 4.865 & 132 & 2.368 .480 \\
\hline Salza (Geekguy) & César & 15.962 & 81 & 384.690 \\
\hline San Juan & Valentí & 167.628 & 106 & 13.244 .513 \\
\hline Santos (Chicageek) & Elena & 32.089 & 217 & 2.961 .683 \\
\hline Senabre & Dani & 18.306 & 78 & 958.515 \\
\hline
\end{tabular}


lo, y sólo un 7,8\% lanza preguntas. Ese aislamiento afecta también a la escasa aplicación realizada de las estrategias de convergencia mediática. Sólo en un 4,3\% de los vídeos analizados se mencionan las redes sociales del periodista, siendo un porcentaje similar el de aquellos que aluden al medio para el que trabajan, y sólo un $3 \%$ invita a visitar o consumir otro producto periodístico.

La principal intencionalidad de los vídeos es la promoción propia $(38,2 \%)$ y la promoción de un producto $(29,4 \%)$, reduciéndose al 2,9\% cuando el objetivo es criticar una información.

\subsection{Técnicamente, correctos}

Desde la perspectiva técnica se observa que el 39,2\% de los vídeos hace uso de una edición básica al corte, aunque un $24,5 \%$ recurre a una edición más compleja con algunos toques de postproducción. Casi un 7,8\% son vídeos de una única toma, y el 3,9\% grabaciones en bruto.

El sonido se fundamenta en el obtenido de la fuente de grabación (53,9\%) aunque 3 de cada 10 vídeos también recurren a fuentes externas de grabación. Son excepcionales los casos con volumen inadecuado y normalmente se justifica por invasión del sonido ambiente. También es habitual añadir música en la edición (41,2\%), frente a un 29,4\% que la evita.

Las grabaciones analizadas tampoco consideran que el $72 \%$ de los usuarios accede a YouTube desde un smartphone, y un $62 \%$ desde una tableta o el ordenador (IAB Spain, 2017). Pese a este consumo predominante en pantallas pequeñas los periodistas utilizan equilibradamente primeros planos, planos medios y generales $(22,5 \%)$, combinación de plano medio y primeros (17,6\%); el uso exclusivo de primer plano se reduce al 5,9\% de las piezas analizadas.

Analizando la iluminación existe un buen equilibro en el uso de luz artificial (27,5\%) y luz natural (18,6\%), si bien lo normal es combinar ambas. Sólo un 2,9\% de vídeos están insuficientemente iluminados.

Se puede concluir por tanto que el nivel técnico aplicado es correcto. Un análisis de contenido pionero a 882 vídeos informativos publicados en YouTube (Peer; Ksiazek, 2010) reveló que la calidad técnica de la imagen y el sonido contrastaba con una ausencia de estándares básicos periodísticos (carencia de fuentes, escasa precisión), y que este contenido relajado que se desviaba del ofrecido habitualmente por la televisión, era reconocido por las audiencias otorgándole un amplio volumen de visitas $y$, por lo tanto, popularidad.

Desde la perspectiva semántica, los títulos son mayoritariamente descriptivos $(56,9 \%)$, aunque también hay titulares directos $(21,6 \%)$, de gancho $(17,6 \%)$ y algunos instructivos $(3,9 \%)$. En las descripciones predominan textos descriptivos con enlaces a fuentes del propio autor. Las descripciones que incluyen enlaces a fuentes externas son un $6 \%$.

En las reacciones de los usuarios a los vídeos (tabla 5), siempre predominan las positivas, y quienes mejores resultados consiguen son dos de los perfiles más jóvenes, Dani Senabre y Valentí San Juan, así como el periodista televisivo Iker Jiménez.

Tabla 5. Reacciones a los vídeos de los periodistas destacados

\begin{tabular}{|l|r|r|c|}
\hline \multicolumn{1}{|c|}{ Youtuber } & Reproducciones & Me gusta & No me gusta \\
\hline Arráez, David & 39.515 & 413 & 30 \\
\hline Cabrera, Andrés & 199.025 & 6.099 & 135 \\
\hline Calabor, Luis & 2.481 .909 & 2.265 & 386 \\
\hline Casquet, Noemí & 651.882 & 5.960 & 271 \\
\hline Durán Escabias, Imanol & 380.858 & 1.224 & 103 \\
\hline Fáez, Rodrigo & 214.182 & 7.913 & 225 \\
\hline Gago, Manuel & 48.964 & 479 & 10 \\
\hline Gómez, Lourdes & 7.976 & 257 & 9 \\
\hline Jiménez, Iker & 456.291 & 14.232 & 624 \\
\hline Manfredi, Antonio & 39.751 & 22 & 7 \\
\hline Quevedo, Luis & 828.872 & 456 & 61 \\
\hline Salza, César (Geekguy) & 138.986 & 1.880 & 253 \\
\hline San Juan, Valentí & 1.860 .870 & 28.236 & 873 \\
\hline Santos (Chicageek), Elena & 576.494 & 2.288 & 304 \\
\hline Senabre, Dani & 260.533 & 12.699 & 211 \\
\hline
\end{tabular}

\section{Conclusiones}

El cambio es una constante en la historia del periodismo, pero esta investigación demuestra que la iniciativa audiovisual es un reto pendiente para la mayoría de los periodistas. Según el Reuters Institute, YouTube es la segunda red social del mundo más utilizada para el consumo de noticias, pero para los periodistas representa aún un territorio por conquistar. A pesar de ello, la novedad del presente estudio exploratorio radica en construir el primer directorio de periodistas españoles creadores de un canal independiente en la plataforma adquirida por Google un año después de su lanzamiento.

Las actividades diversas a las que puede dedicarse un periodista hace difícil establecer cuántos ejercen la profesión en España, aunque de los 7.137 periodistas registrados por el Servicio Público de Empleo Estatal (SPEE) esta investigación muestra cómo sólo 237 tienen un perfil público en YouTube, cifra que se reduce a 36 al seleccionar los perfiles de éxito. La oferta creada resulta híbrida, procede de perfiles ambiguos interesados por la experimentación, y donde periodísticamente destaca la producción especializada. Algunas de estas iniciativas profesionales independientes que surgen sin 
estar vinculadas a un medio llegan a obtener en ocasiones reproducciones que estarían por encima de muchos programas emitidos por la televisión convencional, aunque no existe una correlación cuantitativa entre el número de vídeos producidos, los suscriptores y las reproducciones obtenidas. Pero éstos son resultados excepcionales, ya que predominan las cuentas que ignoran, alteran o rediseñan su actividad profesional habitual.

Estos datos confirman que los periodistas españoles infrautilizan YouTube al no implementar estrategias dirigidas a la creación de marca personal, de refuerzo de su reputación digital o de incremento de su autoridad profesional, desaprovechando por tanto la oportunidad de aproximarse a nuevos públicos, de explorar los nuevos límites de la profesión (Carlsson; Lewis, 2015), mejorar sus opciones de empleabilidad (Marauri-Castillo; Cantalapiedra-González; Álvarez-Fernández, 2018) o incluso aportar transparencia a su actividad para frenar la difusión de desinformación.

Esta reducida participación puede estar ligada a tres aspectos fundamentalmente:

- la cultura periodística, que tradicionalmente toma distancia del acontecimiento para asegurar la objetividad;

- la ausencia de una misión informativa en los orígenes de YouTube, dando prioridad a contenidos superficiales, caseros, $\mathrm{y}$ al infotainment;

- aunque el nivel de autonomía y transparencia en el entorno laboral son síntomas de independencia (Deuze, 2005), en un escenario inestable estas acciones representan simultáneamente un riesgo.

Consciente de este panorama, en diciembre de 2018 Google News Initiative anunció la financiación de 87 proyectos de medios de 23 países con 25 millones de dólares para innovar en el vídeo online y el videoperiodismo. Europa Press, uno de los beneficiarios de la iniciativa, creará una red de videoperiodistas móviles (Katz, 2018), lo que permitirá detectar si el respaldo económico resulta clave para aumentar el emprendimiento periodístico audiovisual.

Por ello, esta investigación propone abrir futuras líneas relacionadas con la motivación del profesional para estar presente o ausente de YouTube, que permitan conocer las expectativas que proyecta sobre sus acciones en la plataforma, o incluso a la percepción y la experiencia del usuario tras el consumo de estos contenidos.

\section{Referencias}

Amoedo, Avelino; Vara-Miguel, Alfonso; Negredo, Alfonso (2018). Digital news report.es 2018. Una audiencia diversa y preocupada por la desinformación. Universidad de Navarra. https://drive.google.com/file/d/1_MqxpbPvMQM1lpvjsGm4QOKxIMC8IZ_D/view

Antony, Mary-Grace; Thomas, Ryan J. (2010). "This is citizen journalism at its finest': YouTube and the public sphere in the Oscar Grant shooting incident". New media \& society, v. 12, n. 8, pp. 1280-1296.

https://doi.org/10.1177/1461444810362492

Banikarim, Susie (2017). "R.I.P. Pivot to video (2017-2017)". Nieman Lab. http://www.niemanlab.org/2017/12/r-i-p-pivot-to-video-2017-2017/

Benítez-de-Gracia, María-José; Herrera-Damas, Susana (2018). “El reportaje inmersivo en vídeo 360: diseño de un modelo de análisis". El profesional de la información, v. 27, n. 1, pp. 149-161.

https://doi.org/10.3145/epi.2018.ene.14

Berrocal-Gonzalo, Salomé; Martín-Jiménez, Virginia; Gil-Torres, Alicia (2017). "Líderes políticos en YouTube: información y politainment en las elecciones generales de 2016 (26J) en España”. El profesional de la información, v. 26, n. 5, pp. 937-946.

https://doi.org/10.3145/epi.2017.sep.15

Bossio, Diana; Sacco, Vittoria (2017). "From 'selfies' to breaking tweets". Journalism practice, v. 11, n. 5, pp. 527-543. https://doi.org/10.1080/17512786.2016.1175314

Campos-Freire, Francisco; Rúas-Araújo, José; López-García, Xosé; Martínez-Fernández, Valentín-Alejandro (2016). "Impacto de las redes sociales en el periodismo". El profesional de la información, v. 25, n. 3, pp. 449-457. https://doi.org/10.3145/epi.2016.may.15

Carlson, Matt; Lewis, Seth C. (eds.) (2015). Boundaries of journalism: Professionalism, practices and participation. Londres: Routledge. ISBN: 9781138020672

Chen, Chih-Ping (2013). “Exploring personal branding on YouTube”. Journal of internet commerce, v. 12, n. 4, pp. 332347.

https://doi.org/10.1080/15332861.2013.859041

Chi, Clifford (2018). "How long should your videos be? Ideal lengths for Facebook, Instagram, Twitter, and YouTube [Infographic]". HubSpot.

https://blog.hubspot.com/marketing/how-long-should-videos-be-on-instagram-twitter-facebook-youtube 
De-Aguilera-Moyano, Miguel; Castro-Higueras, Antonio; Pérez-Rufí, José-Patricio (2019). “Between broadcast yourself and broadcast whatever: YouTube's homepage as a synthesis of its business strategy". El profesional de la información, v. 28, n. 2, e280206.

https://doi.org/10.3145/epi.2019.mar.06

Deuze, Mark (2005). "What is journalism?: Professional identity and ideology of journalists reconsidered". Journalism, v. 6 , n. 4 , pp. 442-464.

https://doi.org/10.1177/1464884905056815

EGM (2012). Audiencia de internet. Abril/mayo de 2012. AIMC.

https://www.aimc.es/a1mc-cOnt3nt/uploads/2012/07/internet212.pdf

EGM (2018). Audiencia de internet. Abril/mayo 2018. AIMC.

https://www.aimc.es/a1mc-cOnt3nt/uploads/2018/06/internet218.pdf

Ericsson Consumerlab (2017). TV and media 2017. A consumer-driven future of media.

https://www.ericsson.com/en/trends-and-insights/consumerlab/consumer-insights/reports/tv-and-media-2017

Ford, Heather; Wajcman, Judy (2017). “'Anyone can edit', not everyone does: Wikipedia's infraestructure and the gender gap". Social studies of science, v. 47, n. 4, pp. 511-527.

https://doi.org/10.1177/0306312717692172

García-Rapp, Florencia (2016). "The digital media phenomenon of YouTube beauty gurus: the case of Bubzbeauty". International journal of web based communities, v. 12, n. 4, pp. 360-375.

https://doi.org/10.1504/IJWBC.2016.080810

Hanusch, Folker; Bruns, Axel (2017). "Journalistic branding on Twitter”. Digital journalism, v. 5, n. 1, pp. $26-43$.

https://doi.org/10.1080/21670811.2016.1152161

Harbers, Frank (2016). "Time to engage". Digital journalism, v. 4, n. 4, pp. 494-511.

https://doi.org/10.1080/21670811.2015.1124726

Holton, Avery E.; Molyneux, Logan (2017). "Identity lost? The personal impact of brand journalism”. Journalism, v. 18, n. 2, pp. 195-210.

https://doi.org/10.1177/1464884915608816

IAB Spain (2017). Estudio anual de redes sociales 2017. IAB Spain.

https://iabspain.es/wp-content/uploads/iab_estudioredessociales_2017_vreducida.pdf

Ireton, Cherilyn; Posetti, Julie (coords.) (2018). Journalism, 'fake news' and disinformation. Paris: Unesco. ISBN: 97892 31002816

https://en.unesco.org/fightfakenews

Jackson, Jasper (2016). "Facebook live video service sees company paying news publishers". The guardian, 6 Apr. https://www.theguardian.com/media/2016/apr/06/facebook-live-video-paying-news-publishers-buzzfeed

Kalogeropoulos, Antonis (2017). “Online news video consumption”. Digital journalism, v. 6, n. 5, pp. $651-665$. https://doi.org/10.1080/21670811.2017.1320197

Katz, Tim (2018). "GNI, fondo para la innovación en YouTube: apoyo a 87 medios de comunicación para desarrollar el videoperiodismo". Blog oficial de Google España, 17 diciembre.

https://espana.googleblog.com/2018/12/gni-fondo-para-la-innovacion-en-youtube.html

Lapowsky, Issie (2018). "YouTube debuts plan to promote and fund 'authoritative' news". Wired, 7 Sept.

https://www.wired.com/story/youtube-debuts-plan-to-promote-fund-authoritative-news

Lewis, Seth C. (2012). "The tension between professional control and open participation". Information, communication \& society, v. 15, n. 6, pp. 836-866.

https://doi.org/10.1080/1369118X.2012.674150

Lorenz, Taylor (2018). "Personal branding is more powerful than ever". Nieman Lab. http://www.niemanlab.org/2018/12/personal-branding-is-more-powerful-than-ever

Marauri-Castillo, Íñigo; Cantalapiedra-González, María-José; Álvarez-Fernández, Carmen (2018). “Blog y Twitter, la combinación perfecta del comunicador digital: los casos de Escolar.net, El comidista y Mi mesa cojea". El profesional de la información, v. 27, n. 2, pp. 349-358.

https://doi.org/10.3145/epi.2018.mar.13

Masip, Pere (2016). "Investigar el periodismo desde la perspectiva de las audiencias". El profesional de la información, v. 25, n. 3, pp. 323-330.

https://doi.org/10.3145/epi.2016.may.01 
Matsa, Katerina-Eva; Silver, Laura; Shearer, Elisa; Walker, Mason (2018). Western Europeans under 30 view news media less positively, rely more on digital platforms than older adults. Pew Research Center. http://www.journalism.org/wp-content/uploads/sites/8/2018/10/PJ_2018.10.30_europe-age_FINAL3.pdf

Molyneux, Logan (2014). "What journalists retweet: Opinion, humor, and brand development on Twitter". Journalism, v. 16, n. 7, pp. 920-935. https://doi.org/10.1177/1464884914550135

Molyneux, Logan; Lewis, Seth C.; Holton, Avery E. (2018). “Media work, identity, and the motivations that shape branding practices among journalists: An explanatory framework". New media \& society, v. 21, n. 4. https://doi.org/10.1177/1461444818809392

Noguera-Vivo, José-Manuel (2016). “Metamedios y periodismo: revisión panorámica de los nuevos cibermedios". El profesional de la información, v. 25, n. 3, pp. 341-350.

https://doi.org/10.3145/epi.2016.may.03

Origgi, Gloria (2018). "Say goodbye to the information age: it's all about reputation now". Aeon. https://aeon.co/ideas/say-goodbye-to-the-information-age-its-all-about-reputation-now

Palomo, Bella; Palau-Sampio, Dolors (2016). “El periodista adaptativo. Consultores y directores de innovación analizan las cualidades del profesional de la comunicación”. El profesional de la información, v. 25, n. 2, pp. 188-195. https://doi.org/10.3145/epi.2016.mar.05

Paulussen, Steve; Harder, Raymond-A. (2014). "Social media references in newspapers. Facebook, Twitter and YouTube". Journalism practice, v. 8, n. 5, pp. 542-551. https://doi.org/10.1080/17512786.2014.894327

Peer, Limor; Ksiazek, Thomas (2010). "YouTube and the challenge to journalism”. Journalism studies, v. 12, n. 1, pp. $45-63$. https://doi.org/10.1080/1461670X.2010.511951

Reinemann, Carsten; Stanyer, James; Scherr, Sebastian; Legnante, Guido (2011). "Hard and soft news: A review of concepts, operationalizations and key findings". Journalism, v. 13, n. 2, pp. 221-239.

https://doi.org/10.1177/1464884911427803

Røssland, Lars-Arve (2007). "The professionalization of the intolerable. Popular crime journalism in Norway". Journalism studies, v. 8, n. 1, pp. 137-152.

https://doi.org/10.1080/14616700601056908

Serrano-Cobos, Jorge (2016). "Tendencias tecnológicas en internet: hacia un cambio de paradigma". El profesional de la información, v. 25, n. 6, pp. 843-850.

https://doi.org/10.3145/epi.2016.nov.01

Shaw, Aaron; Hargittai, Eszter (2018). "The pipeline of online participation inequalities: The case of Wikipedia editing". Journal of communication, v. 68, n. 1, pp. 143-168.

https://doi.org/10.1093/joc/jqx003

Singer, Jean B. (2014). "Getting past the future: Journalism ethics, innovation, and a call for 'flexible first'”. Comunicação e sociedade, v. 25, pp. 67-82.

https://doi.org/10.17231/comsoc.25(2014).1860

Smith, Aaron; Anderson, Monica (2018). Social media use in 2018. Pew Research Center. http://assets.pewresearch.org/wp-content/uploads/sites/14/2018/03/01105133/PI_2018.03.01_Social-Media_FINAL.pdf

Tandoc, Edson C.; Vos, Tim P. (2015). "The journalist is marketing the news. Social media in the gatekeeping process". Journalism practice, v. 10, n. 8, pp. 950-966. https://doi.org/10.1080/17512786.2015.1087811

Wardle, Claire; Derakhshan, Hossein (2017). Information disorder: Toward an interdisciplinary framework for research and policy making. Council of Europe Report.

https://rm.coe.int/information-disorder-toward-an-interdisciplinary-framework-for-researc/168076277c 